\title{
Paniculitis mesentérica asociada con absceso intraabdominal ${ }^{*}$ \\ Mesenteric panniculitis with intraabdominal abcess. Report of one case
}

\begin{abstract}
Drs. GERMÁN VISCIDO', DANIEL NAPOLITANO', GUILLERMO RIVOIRA', MATÍAS PARODI', MARTÍN BAROTTO', HÉCTOR PICÓN' ${ }^{1}$, MARCELO DONIQUIAN' ${ }^{1}$, RAFAEL PALENCIA'.
\end{abstract}

${ }^{1}$ Clínica Universitaria Privada Reina Fabiola, Córdoba, Argentina.

\section{RESUMEN}

La paniculitis mesentérica es una enfermedad inflamatoria del tejido adiposo del mesenterio, de presentación infrecuente y que se caracteriza, por el engrosamiento, endurecimiento y nodularidad del mismo. Se pone en consideración un caso clínico en un varón joven, con una forma de presentación poco común que requirió de laparotomía exploradora para realizar el diagnóstico y tratamiento.

PALABRAS CLAVE: Paniculitis mesentérica, mesenteritis, lipodistrofia.

\begin{abstract}
We report a 28 years old obese male presenting with abdominal pain of increasing intensity lasting 15 days. In the last days, diarrhea and fever appeared. On abdominal examination a $8 \mathrm{~cm}$ painful mass was palpated. An abdominal ultrasound showed an anechoic mass in the epigastrium. An abdominal CAT scan showed an inflammatory mass that involves the small bowel. The patient is subjected to a percutaneous needle aspiration, obtaining $50 \mathrm{ml}$ of a purulent fluid. Due to persistence of fever, the patient was operated finding an inflammation that involved the mesentery and the great omentum and covering abscess. The pathologic study of the surgical piece reported a unspecific nodular mesenteric panniculitis
\end{abstract}

KEY WORDS: Mesenteric panniculitis, abscess.

\footnotetext{
* Recibido el 17 de Enero de 2008 y aceptado para publicación el 27 de Febrero del 2008.

Correspondencia: Dr. Germán R. Viscido

Derqui 312 Piso 6ํㅡ, Ciudad de Córdoba, Argentina

E-mail: germanviscido@hotmail.com
} 


\section{INTRODUCCIÓN}

La paniculitis mesentérica, es una inflamación inespecífica del tejido adiposo mesentérico ${ }^{1-3}$. Fue descripta por primera vez en 1955 y es una rara entidad que ocurre en la adultez tardía ${ }^{1}$. Cursa en general en forma asintomática y en pocas ocasiones se presenta con sintomatología clínica, siendo un hallazgo frecuente y casual al realizar exploraciones radiológicas abdominales ${ }^{4}$. Rara vez requiere tratamiento y la resección o derivación intestinal sólo están indicadas en la obstrucción intestinal ${ }^{5}$.

\section{Descripción del caso}

Paciente masculino de 28 años de edad, con antecedentes de obesidad. Consulta en guardia refiriendo dolor abdominal de 15 días de evolución, de comienzo gradual, inicialmente de intensidad leve, incrementándose paulatinamente con el correr de los días hasta ser moderado; de característica continua, con exacerbaciones cólicas episódicas, localizado en epigastrio y región periumbilical, sin irradiación.

Además presenta náuseas sin vómitos en las últimas 48 horas. Cambios en el hábito intestinal de 5 días de evolución, manifestando 3 deposiciones diarreicas por día, acuosas sin moco, sangre ni pus.

Dos días previos presentó episodios de fiebre de $38^{\circ}$ acompañados de escalofríos.

No refiere antecedentes de similares características.

Examen físico: febril $38,3^{\circ} \mathrm{C}$, taquicárdico 105 latidos por minuto, taquipneico 18 respiraciones por minuto.

Se observa un abdomen globuloso, no distendido, simétrico y sin cicatrices quirúrgicas. A la palpación blando, depresible con dolor, defensa y signos de irritación peritoneal en epigastrio, periumbilical y cuadrante superior izquierdo. Se palpa tumoración redondeada, dura, de $8 \mathrm{~cm}$ de diámetro, superficie lisa, inmóvil y dolorosa.

Con diagnóstico de abdomen agudo inflamatorio más tumor abdominal es internado.

Se indican analgésicos y antibióticoterapia empírica con Ampicilina-Sulbactam 1,5 gr c/6h. Previamente se toman muestras de hemo y urocultivo. Exámenes de laboratorio evidencian leucocitosis con desviación a la izquierda y VSG elevada.

Ecografía abdominal: en región epigástrica imagen anecoica de $3,4 \mathrm{~cm} \times 2,9 \mathrm{~cm}$ compatible con absceso interasas.

TC con contraste oral y endovenosa: se visualiza tumoración inflamatoria que compromete el epiplón mayor, en continuidad con el ombligo, com- primiendo el intestino delgado, desplazándolo de su posición normal y con sus paredes engrosadas. La grasa mesentérica se halla enrarecida. El páncreas, vesícula biliar, colon y resto de los órganos son normales.

Debido a estos hallazgos, y con diagnóstico de absceso interasas, se decide realizar punción guiada con ecografía y colocación de drenaje. Se extraen $50 \mathrm{~cm}$ de líquido purulento que es enviado a estudio físicoquímico y bacteriológico, los que informan intensa reacción inflamatoria pero sin hallar gérmenes. Se agrega al esquema antibiótico gentamicina $240 \mathrm{mg} \mathrm{c} / 24 \mathrm{~h}$ y metronidazol $500 \mathrm{mg}$ $\mathrm{c} / 8 \mathrm{~h}$.

A pesar de la instauración del nuevo esquema antibiótico, el paciente persiste febril, taquicárdico, con dolor y distensión abdominal y leucocitosis en aumento, por lo que se decide realizar exploración abdominal debido a falla en el tratamiento mínimamente invasivo.

Se realiza laparotomía exploradora, evidenciándose a nivel inframesocolónico firme proceso inflamatorio que afecta al mesenterio y al epiplón mayor que da cobertura a una cavidad abscedada de la cual emerge líquido purulento. Este proceso inflamatorio comprime las primeras asas yeyunales por lo que se decide resecar el plastrón y colocar un drenaje. No se encontró otra causa abdominal del proceso infeccioso.

La anatomía patológica evidenció una lesión nodular de $17 \times 14 \times 3 \mathrm{~cm}$ de color pardo amarillento. En la microscopía se evidencia tejido fibroadipocitario, que por sectores se destaca importante inflamación crónica granulomatosa, constituida por linfocitos, histiocitos, plasmocitos y células multinucleadas; se destacan áreas de disposición nódulo folicular de linfocitos. Conclusión: Paniculitis Mesentérica Nodular Inespecífica.

El paciente evolucionó en forma favorable, otorgándose el alta a los 6 días.

A casi 2 años, el paciente se encuentra en óptimas condiciones, sin evidencia de patología residual.

\section{DISCUSIÓN}

La paniculitis mesentérica (PM) es un raro desorden caracterizado por la inflamación crónica e inespecífica del tejido adiposo del mesenterio del intestino delgado ${ }^{1,2,3,6}$. Se conoce también como mesenteritis, lipodistrofia aislada, xantogranuloma retroperitoneal, lipogranulomatosis esclerosante, lipogranuloma del mesenterio, manifestación mesentérica de la enfermedad de Weber-Christian y pseudotumor inflamatorio ${ }^{1,2,5}$. El término "Paniculitis Mesentérica" fue acuñado por Odgen en 19607. 
Tabla 1

CLASIFICACIÓN DE LA PANICULITIS MESENTÉRICA SEGÚN MAYO CLINIC

Clasificación de Paniculitis Mesentérica que

compromete al mesenterio del intestino delgado

Tipo 1: Clase más común. Engrosamiento difuso del mesenterio, desde la raíz a los bordes del intestino delgado

Tipo 2: Masa nodular aislada en la raíz del mesenterio

Tipo 3: Mesenterio con múltiples nódulos de distintos tamaños

En las afecciones inflamatorias y fibróticas del mesenterio se describen tres entidades básicas: mesenteritis retráctil, paniculitis mesentérica y fibrosis retroperitoneal. Estas enfermedades podrían representar distintos aspectos del mismo espectro de inflamación y cicatrización de estas estructuras $^{8}$. Emory et al, sobre 84 casos, concluye que la lipodistrofia mesentérica, paniculitis mesentérica y la mesenteritis retráctil, aparentemente representan variantes histológicas de una misma entidad y, en la mayoría de los casos, el término "mesenteritis retráctil" es el más adecuado9. Una de las pocas clasificaciones de esta enfermedad data del año 1974 en la serie de la Clínica Mayo² (Tabla 1).

Aproximadamente 200 casos de esta afección han sido publicados en la literatura ${ }^{3}$. Ocurre en general en la adultez tardía, con una media de 5060 años ${ }^{1,2,10}$; es más común en varones, en una relación hombre-mujer de 1,8:11. Es extremadamente infrecuente en la población pediátrica, quizás por la menor cantidad de grasa mesentérica de los infantes comparados con los adultos ${ }^{2,9}$.

Aunque de patogenia y etiología desconocidas, se han apuntado como posibles agentes etiológicos la isquemia, infecciones, traumatismos abdominales, antecedentes quirúrgicos, fármacos y fenómenos autoinmunes ${ }^{11}$. Durst et $\mathrm{al}^{13}$, relataron la asociación de PM con cirugías recientes en el $17 \%$ de los casos, principalmente apendicectomía y colecistectomía. Se ha relatado también asociada a trombosis mesentérica, arteriopatía mesentérica, drogas, lesiones térmicas, avitaminosis, reacciones de hipersensibilidad y retención del material de sutura ${ }^{2}$. También ha sido relacionada con enfermedades granulomatosas, malignas, pancreatitis y tuberculosis ${ }^{12}$. La posible asociación con enfermedades inflamatorias fibrosantes (como la fibrosis retroperitoneal, colangitis esclerosante, tiroiditis fibrosante de Riedel y el seudotumor orbitario) está bien documentada; asimismo está descripta como síndrome paraneoplásico en el contexto de enfermedades linfoproliferativas, melanomas y neoplasias de mama, colon y pulmón ${ }^{3,4}$. Es infrecuente la asociación de una infección bacteriana con la $\mathrm{PM}^{1}$.

En general, se localiza en el mesenterio del intestino delgado ${ }^{1,5}$, pero también puede afectar el mesocolon transverso, mesocolon sigmoides, epiplón y retroperitoneo menos frecuentemente ${ }^{1,5,11,14}$.

Es un proceso que en pocas situaciones se presenta con sintomatología clínica y suele ser un hallazgo frecuente y casual al practicar exploraciones radiológicas abdominales ${ }^{4}$. Cuando se presentan síntomas, los más frecuentes son dolor abdominal, anorexia, plenitud abdominal y pérdida de peso ${ }^{2,15,16}$; también puede manifestarse con signos y síntomas de oclusión de intestino delgado ${ }^{1,17}$. Otras manifestaciones comprenden estreñimiento, fiebre, diarrea y la palpación de una masa abdominal, la cual se halla en la mitad de los casos ${ }^{5,11}$. Excepcionalmente, se han reportado casos con sangrado rectal, ictericia y obstrucción gástrica ${ }^{2}$. La presentación como abdomen agudo ha sido también publicada ${ }^{18}$. La duración de los síntomas varía desde dos semanas hasta varios años ${ }^{10}$. En nuestro caso, que se presentó como un abdomen agudo inflamatorio con una evolución de 15 días, se evidenció un absceso interasa que no pudo ser resuelto con drenaje percutáneo. No encontramos, en la bibliografía consultada, la descripción de esta patología como una colección purulenta localizada.

Los análisis de laboratorio arrojan, de ordinario, un resultado normal, salvo la velocidad de eritrosedimentación que suele elevarse ${ }^{1,5}$.

Los estudios imagenológicos con contraste baritado del intestino delgado y colon pueden ser normales ${ }^{2} \mathrm{o}$ advertirse un desplazamiento extrínseco de las asas intestinales, asas fijas y dilatadas del intestino delgado y un aspecto especular o aserrado de la mucosa, compatible con una inflamación extrínseca ${ }^{5}$, la pared del colon puede tener aspecto irregular sin lesiones intraluminales ${ }^{19}$.

En la ecografía abdominal puede identificarse una masa hiperecogénica bien definida en la raíz del mesenterio desplazando el intestino. Es posible visualizar la interfaz entre la PM y el tejido normal ${ }^{20}$.

Los hallazgos más característicos en la TC son: aumento de la densidad grasa del mesenterio bien delimitada que provoca un efecto de masa con desplazamiento de las asas intestinales adyacentes, presencia de ganglios mesentéricos menores a $5 \mathrm{~mm}$ y por último, un halo graso o pseudocápsula que rodea a los ganglios y vasos (signo 
del anillo graso o "fat-ring sign"3,21-23. El aspecto de este proceso en la TC es inespecífico y puede ser determinado por un conjunto de otras enfermedades.

La colonoscopía usualmente no revela ninguna alteración puesto que la PM es una afección extrínseca ${ }^{2}$, o en caso de compromiso del colon, pueden encontrarse hallazgos inespecíficos como estenosis o edema de la mucosa rectosigmoidea ${ }^{24}$.

Si bien las técnicas de imágenes son útiles, la biopsia del mesenterio es fundamental para el diagnóstico ${ }^{1,5,11,15}$. Ésta se puede realizar mediante laparotomía o laparoscopía ${ }^{24}$. La combinación de ecografía o TC y punción con aguja fina podrían ayudar a establecer el diagnóstico sin requerir a laparotomía ${ }^{11,26}$. Badiola-Varela et al, sugirieron que la cirugía podría ser evitada si las características de la PM fueron identificadas en $\mathrm{TC}^{27}$.

Histopatológicamente, la enfermedad progresa en tres etapas ${ }^{2}$ :

1. El primer estadío es la Lipodistrofia Mesentérica en la que la grasa mesentérica normal es reemplazada por una capa de macrófagos espumosos; los cambios inflamatorios son mínimos o inexistentes y la enfermedad tiende a ser asintomática con buen pronóstico.

2. La segunda etapa es la Paniculitis Mesentérica, caracterizada histológicamente por un infiltrado con células plasmáticas y pocos leucocitos polimorfonucleares, células gigantes de cuerpo extraño y macrófagos.

3. La etapa final es la Mesenteritis Retráctil, en la que se observan depósitos de colágeno, fibrosis e inflamación. Estos depósitos de colágeno conducen a la retracción del mesenterio con la formación de masas abdominales y síntomas obstructivos.

El diagnóstico diferencial debe ser hecho con las patologías que figuran en la Tabla $2^{2,11,20}$.

\section{Tabla 2 \\ DIAGNÓSTICO DIFERENCIAL DE LA PANICULITIS MESENTÉRICA}

\begin{tabular}{ll}
\hline Lipomas & $\begin{array}{l}\text { Enfermedades por } \\
\text { Micobacterias }\end{array}$ \\
Liposarcoma & Linfangioma \\
Linfoma & Histoplasmosis \\
Tumor desmoide & Sarcoma Retroperitoneal \\
Tumor Carcinoide & Amiloidosis \\
Carcinomatosis Peritoneal & Enfermedad de Whipple \\
Fibrosis Retroperitoneal & Mesoteliomas \\
\hline
\end{tabular}

La evolución es variada pero en general es favorable ${ }^{1,2,5}$, desde pacientes asintomáticos hasta infrecuentes casos fatales ${ }^{28-29}$. Akram et al, en la serie de mayor número de pacientes descripta, concluye que la PM, aunque es un proceso relativamente benigno, puede tener un curso prolongado y debilitante con resultados fatales debido a complicaciones de la propia patología o al tratamiento ${ }^{16}$. En general se resuelve espontáneamente y las masas palpables pueden encontrarse a menudo entre 2 y 11 años luego del diagnóstico². El dolor abdominal continúa o reaparece en un $25 \%$ de los pacientes luego del diagnóstico ${ }^{5}$. Debido a la asociación con enfermedades linfoproliferativas, si durante la presentación clínica o en el seguimiento aparecen síntomas de alarma como pérdida de peso, anemia o sudoración nocturna, debemos investigar para descartar estas patologías ${ }^{16}$. También la presencia durante la evolución de anemia, falla renal e hipercalcemia debe alertar la posibilidad de mieloma múltiple ${ }^{30}$

Los casos sintomáticos requieren tratamiento $y$, para algunos autores, la administración de corticoides solos o asociados a colchicina o inmunodepresores (Azatioprina o Ciclofosfamida) es el tratamiento que mejores resultados ofrece ${ }^{31}$ aunque aún no hay consenso en ello. El uso de corticoides puede reducir la inflamación en caso de que la entidad no progrese a la etapa de fibrosis $^{32}$. Otros reportan la terapia con ciclofosfamida en caso de falla del uso de prednisona ${ }^{2}$.

También ha sido relatado el tratamiento con progesterona inhibiendo la proliferación de fibroblastos $^{33}$. En la numerosa serie de la Mayo Clinic, concluyen que los pacientes sintomáticos se benefician con una combinación de Tamoxifeno y Prednisona ${ }^{16} \mathrm{e}$ intentan una propuesta de algoritmo terapéutico para la PM.

El manejo quirúrgico debe ser reservado sólo para la obstrucción intestinal $\left.\right|^{1,2,4,5,15}$ y la isquemia intestinal ${ }^{4,15}$. No se aconseja la resección total de la masa debido a que usualmente no es posible y no se considera beneficiosa ${ }^{34}$.

\section{REFERENCIAS}

1. Hines O, Ashley S. Lesiones del mesenterio, el epiplón y el retroperitoneo. En Operaciones Abdominales. Vol 1. 10ํㅡㄹición. Editorial Médica Panamericana. 1998. Pag. 655-667.

2. Delgado Plasencia L, Rodríguez Ballester L, LópezTomassetti Fernández E. Mesenteric Panniculitis: Experience in our center. Rev Esp Enferm Dig 2007; 99: 291-297.

3. Daskalogiannaki M, Voloudaki A, Prassopoulos P. CT evaluation of mesenteric panniculitis: prevalence 
and associated diseases. AJR 2000; 174: 427-431.

4. Aranda S, Rivero M, Villar A, Selva A. Paniculitis mesentérica sintomática y sarcoidosis. Med Clin (Barc) 2006; 126: 554-559.

5. Turnage R, Li B, Mc Donald J. Pared Abdominal, Ombligo, Peritoneo, Mesenterios, Epiplones y Retroperitoneo. En Tratado de Cirugía Sabiston. Vol II. 17ª Edición. Editorial Elsevier 2006. Pag 11711197.

6. Béchade D, Durand X, Desramé J, Rambelo A, Corberand D, Baranger B, et al. Etiologic spectrum of mesenteric panniculitis: report of 7 cases. Rev Med Interne 2007; 28: 289-295.

7. Odgen WW, Bradburn DM, Rives JD. Panniculitis of the mesentery. Ann Surg 1960; 151: 659-665.

8. Runyon B, Such J. Peritonitis Quirúrgica y otras Enfermedades del Peritoneo, el Mesenterio, el Epiplón y el Diafragma. En Enfermedades Gastrointestinales y Hepáticas, Sleisenger \& Fordtran. Tomo 2. 7a Edición. Editorial Panamericana 2004. Pag 2513-2526.

9. Emory TS, Monihan JM, Carr NJ, Sobón LH. Sclerosing Mesenteritis, Mesenteric Panniculitis and Mesenteric Lipodistrophy: a single entity? Am J Surg Pathol 1997; 2: 392-398.

10. Bashir MS, Abbot CR. Mesenteric Lipodistrophy. J Clin Pathol 1993; 46: 872-874.

11. Colomer Rubio E, Blanes Gallego A, Carbonell Biot C, Villar Grimalt A, Tomás Ivorra H, Llamusi Lorente A. Paniculitis mesentérica con afectación retroperitoneal resuelta tras tratamiento con pulsos de ciclofosfamida endovenosa. An Med Interna 2003; 20: 31-33.

12. Ege E, Akman H, Cakiroglu G. Mesenteric panniculitis associated with abdominal tuberculous lymphadenitis: a case report and review of the literature. The British Journal of Radiology 2002; 75: 378-380.

13. Durst AL, Freund H, Rosenmann E, Birnbaun D. Mesenteric panniculitis: review of the literature and presentation of cases. Surgery 1977; 81: 203-211.

14. Adachi $\mathrm{Y}$, Mori $\mathrm{M}$, Enjoji $\mathrm{M}$, Ueo $\mathrm{H}$, Sugimachi $\mathrm{K}$. Mesenteric panniculitis of the colon. Review of the literature and report of two cases. Dis Colon Rectum 1987; 30: 962-966.

15. Piessen G, Mariette C, Triboulet JP. Mesenteric panniculitis. Ann Chir 2006; 131: 85-90.

16. Akram S, Pardi D, Schaffner J,Smirk T. Sclerosing Mesenteritis: Clinical Features, and Outcomes in Nine-Two Patients. Clinical Gastroenterology and Hepatology 2007; 5: 589-596.

17. Gironés Vila J, Alcobilla Ferrara E, Codina Barrera A, Gómez Castella F, Farres Coll R, Masvidal Calpe $\mathrm{R}$, et al. Mesenteritis retráctil. A propósito de tres casos. Rev Esp Enf Digest 1995; 87: 62-64.

18. Shah AN, You $\mathrm{CH}$. Mesenteric lipodystrophy presenting as an acute abdomen. Southern Med J 1982; 75: 1025-1026.

19. Patel N, Saleeb S, Teplick S. Cases of the day. RadioGraphics 1999; 19: 1083-1085.

20. Rosón N, Garriga V, Cuadrado M, Pruna X, Carbó
S, Vizcaya S, et al. Sonographic findings of mesenteric panniculitis: correlation with CT and literature review. J Clin Ultrasound 2006; 34: 169176.

21. Raichholz G, Gambra S, Staffieri R, Oxilia H. Paniculitis mesentérica: Hallazgos en tomografía computada y enfermedades asociadas. Anuario Fundación Dr. J. R. Villavicencio 2006; 16: 72-76.

22. Varela C, Fuentes M, Rivadeneira R. Procesos inflamatorios del tejido adiposo intrabdominal, causa no quirúrgica de dolor abdominal: hallazgos en tomografía computada. Rev Chil Radiol 2004; 10: 28-34.

23. Lawler LP, McCarthy DM, Fishman EK, Hruban R. Sclerosing mesenteritis: depiction by multidetector $\mathrm{CT}$ and three-dimensional volume rendering. Am J Roentgenol 2002; 178: 97-99.

24. Seo M, Okada M, Okina S, Ohdera K, Nakashima $\mathrm{R}$, Sakisaka S. Mesenteric panniculitis of the colon with obstruction of the inferior mesenteric vein: report of a case. Dis Colon Rectum 2001; 44: 885889.

25. Espinosa MD, Anguita F, Nogueras F, Castro T, De Teresa FJ, Martín Vivaldi R. Paniculitis mesentérica: diagnóstico laparoscópico. Rev Esp Enferm Digest 1997; 89: 791-799.

26. Janisch HD, von Kleist D, Pfretzschner C, Székessy T, Pfannkuch F, Bauer FE. Mesenteric lipoystrophy: differential diagnosis of a benign abdominal tumor. Wien Med Wochenschr 1984; 134: 417-419.

27. Badiola-Varela CM, Sussman SK, Glickstein MF. Mesenteric panniculitis: findings on CT, MRI, and angiography. Case report. Clin Imaging. 1991; 15: 265-267.

28. Caballero Oliver A, Madrid M, Gutiérrez B, Borrero M, Calderón S. Paniculitis mesentérica: un caso de presentación aguda y fatal? An Med Interna 1995; 12: 36-38.

29. Andersen JA, Rasmussen NR, Pedersen JK. Mesenteric panniculitis: a fatal case. Am J Gastroenterol 1982; 77: 523-525.

30. Goh J, Otridge B, Brady H, Breatnach E, Dervan P, MacMathuna P. Aggressive multiple myeloma presenting as mesenteric panniculitis. Am J Gastroenterol 2001; 96: 238-241.

31. Genereau T, Bellin MF, Wechsler B, Le TH, Bellanger $\mathrm{J}$, Grellet J, et al. Demonstration of efficacy of combining corticosteroids and colchicine in two patients with idiopathic sclerosing mesenteritis. Dig Dis Sci 1996; 41: 684-688.

32. Kikiros CS, Edis AJ. Mesenteric panniculitis resulting in bowel obstruction: response to steroids. Aust NZJ Surg 1989; 59: 287-290.

33. Mazure R, Fernández Marty P, Niveloni S, Pedreira $S$, Vazquez H, Smecuol E, et al. Successful treatment of retractile mesenteritis with oral progesterone. Gastroenterology 1998; 114: 1313-1317.

34. Farran L, Ballesta C, Deirós M, Bastida X, Biondo $\mathrm{S}$, Bettónica $\mathrm{C}$, et al. Medical treatment of sclerosing mesenteritis secondary to abdominal surgery. Rev Esp Enferm Dig 2001; 93: 189-190. 